\title{
A review of the safety of vaccines used in routine immunization in Africa
}

\author{
Peter Yamoah ${ }^{1,2}$, Varsha Bangalee ${ }^{2}$, Frasia Oosthuizen ${ }^{2}$
}

1. School of Pharmacy, University of Health and Allied Sciences, Ghana

2. College of Health Sciences, University of KwaZulu Natal, Durban, South Africa

\begin{abstract}
Background: Despite the significant role played by vaccines in global health, concerns over vaccine safety have increased tremendously over the years. There have been occasions where vaccines have caused rare, adverse reactions some of which have led to hospitalizations and even death. It is therefore important to establish the safety profile of routinely used vaccines in order to allay fears pertaining to their use.

Objectives: This review was aimed at pooling together the safety data of selected vaccines used for routine immunization in Africa, a region of the world with paucity of vaccine safety data.

Methods: Adverse Events Following Immunization safety data was searched for rotavirus, yellow fever, measles, rubella, tuberculosis (Bacillus Calmette Guerin-BCG), pneumococcal, Haemophilus Influenza type b, polio, meningococcal and the influenza A (H1N1) vaccines in PUBMED, Google Scholar, Clinical trials.gov and Cochrane controlled register of trials databases.

Results: A total of twenty-four serious AEFIs and twenty-three minor AEFIs were identified from the review. The strength of association between AEFIs and vaccine was high for tuberculosis vaccine and moderate for all other vaccines.

Conclusion: Even though AEFIs (including mild and severe) were identified in the review, all the vaccines studied were generally well tolerated.

Keywords: Safety of vaccines, immunization, Africa.

DOI: https://dx.doi.org/10.4314/ahs.v20i1.28

Cite as: Yamoah P, Bangalee V, Oosthuizen F. A review of the safety of vaccines used in routine immunization in Africa. Afri Health Sci. 2020;20(1):227-37. https://dx.doi.org/10.4314/abs.v20i1.28
\end{abstract}

\section{Introduction}

Vaccines are amongst the top life-saving interventions developed in the history of mankind preventing millions of diseases and deaths annually ${ }^{1}$. The elimination of the deadly diseases, smallpox and rinderpest for instance became possible because of the development and usage of their respective vaccines on a large scale across the globe $^{2-4}$.

Despite the significant role vaccines play in global health, concerns over their safety have increased tremendously over the years ${ }^{5}$. It is important for the general population to be assured that vaccines are less likely to cause

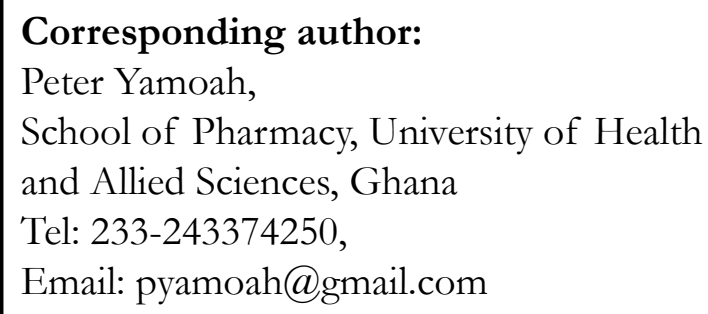

fatalities than the infectious diseases they are meant to prevent and thus generally safe for use. This notwithstanding, there have been occasions where vaccines have caused rare, adverse reactions some of which have led to hospitalizations and even death ${ }^{6}$. This has caused a section of the public to believe that vaccines are harmful, posing a threat to global health as it is a major cause of low turnout in mass vaccination campaigns. During the United States of America multi-state measles outbreak of 2014-2015 for instance, several infected persons were not vaccinated against measles or had unknown vaccination status because of false alarms about fatalities caused by measles containing vaccines which they had read from various websites and print media ${ }^{7-9}$. These fears need to be allayed through educating and adequately communicating the safety profile of vaccines- a very important aspect of vaccine pharmacovigilance. As infectious diseases such as Ebola virus disease continue to emerge and populations continue to increase particularly in poor recited. 
gions like Africa, the use of vaccines will increase proportionally and hence the need to intensify measures to ensure their safety ${ }^{10}$. This calls for commitment towards ensuring good vaccine pharmacovigilance practices by all stakeholders including but not limited to medicine regulators, vaccine marketing authorization holders, healthcare professionals, policy makers, public health practitioners and academic researchers. Vaccine pharmacovigilance has been defined as the science and activities relating to the detection, assessment, understanding and communication of adverse events following immunization (AEFI) and other vaccine and immunization related issues and the prevention of the untoward effects of the vaccine or immunization ${ }^{11}$. One way of improving vaccine safety is the identification and prompt reporting of the adverse events associated with their use to regulatory bodies as the pattern of AEFIs of a particular vaccine product helps to trace the possible cause of the AEFI.

The WHO/CIOMS classify AEFIs under 5 possible causes and these include vaccine product related reactions (e.g. extensive limb swelling following DTP vaccination), vaccine quality related reactions (e.g. failure of the manufacturer to completely inactivate a virus used in the vaccine manufacture), immunization error related reactions (e.g. transmission of infection by contaminated multi-dose vial), immunization anxiety related reactions (e.g. vasovagal syncope) and coincidental events (e.g. fever after vaccination resulting from a co-existing infection like malaria) $)^{12,13}$. AEFIs are classified as serious if they lead to death, hospitalization or prolongation of existing hospitalization, persistent or significant disability or incapacity, congenital anomaly or life threatening event ${ }^{12}$. Minor vaccine reactions usually occur within a few hours of injection and resolve after a short period of time posing little or no danger. These may include pain, swelling and redness at the site of injection whereas minor systemic reactions may include fever, malaise, muscle pain, headache and loss of appetite ${ }^{12}$. It is important to carry out causality assessment of rare but serious AEFIs with a vaccine product because most AEFIs are coincidental events that are falsely attributed to vaccines due to possible temporal associations ${ }^{14}$. Temporal association reports could help future researchers to conduct studies such as controlled clinical trials to generate supportive data for a causal hypothesis ${ }^{15}$. An AEFI investigation may lead to the temporal suspension of a vaccination exercise pending investigation outcome. An AEFI investiga- tion outcome could either recommend the continuation of the vaccination exercise or the outright suspension of vaccination exercise. Vaccine manufacturers must always assess all batches of vaccnes to ensure that good manufacturing practice principles have been duly followed. Most AEFIs resulting from bad manufacturing practices have largely occurred during the introduction of new vaccines for outbreaks which need quick interventions to avoid the rapid spread of disease. Examples of such AEFIs include Bell's palsy resulting from the inactivated nasal flu vaccine and intussusception from the rotavirus vaccine, Rotashield ${ }^{16,17}$. To avoid these AEFIs or reduce their prevalence to the barest minimum, vaccines need to be rigorously monitored for AEFIs especially when they are newly introduced, despite their potential for infection prevention.

Immunization errors, such as the wrong medicine and the wrong route of administration by healthcare professionals have also contributed to AEFIs. A classic example is the wrongful administration of insulin instead of diphtheria, pertussis and tetanus (DTP) vaccine to 70 infants resulting in 21 deaths $^{18}$.

Africa is amongst the poorest regions of the world where large doses of vaccines are administered due to the high burden of infectious diseases which require vaccination as a preventive measure. Evidence in the literature suggests that aside from mass vaccination campaigns, there are many late stage vaccine clinical trials being conducted in Africa ${ }^{19,20}$. However, there is paucity of synthesized body of evidence on the safety of the vaccines used in routine and mass vaccination programs in Africa. Safety data from vaccination campaigns and clinical trials are a good source of information for synthesizing a body of evidence on vaccine safety. This review therefore aimed at pooling together the safety data of selected vaccines used for routine vaccinations using mass vaccination and vaccine clinical trial studies conducted in Africa. In achieving this aim, the types of AEFI identified in vaccine trials and mass vaccination campaigns and the strength of association between the AEFIs and the selected vaccines were assessed. The evidence generated from this study will serve as a baseline for future researchers in vaccine safety and stimulate other researchers to investigate the safety profile of other vaccines not studied in this review. It could also serve as a guide to vaccine pharmacovigilance experts and expanded program on immunization managers as to the likely AEFIs to expect during routine 
and mass vaccinations thereby contributing to improving vaccine safety in Africa.

\section{Methods}

\section{Vaccine information source}

The Centres for Disease Control and Prevention (CDC) website was searched for approved common vaccines for routine immunization in the United States of America and other regions of the world. Out of the twenty-six approved vaccines on the CDC website, seventeen were routinely used in vaccinations in Africa ${ }^{21}$. An initial literature search revealed that nine out of these seventeen vaccines were extensively used in Africa. These included rotavirus, yellow fever, pneumococcal, measles, rubella, tuberculosis (BCG), Haemophilus Influenza type b (Hib), polio and meningococcal vaccines. Further literature search showed that the influenza A (H1N1) vaccine has also been used in many countries in Africa even though not published on the CDC website and was therefore added to the nine.

\section{Eligibility criteria}

\section{Inclusion/exclusion criteria}

The studies included in the database search were phase 1 clinical trials, phase 2 and 3 controlled clinical trials and studies on mass vaccination campaigns conducted in Africa. Information on vaccine AEFIs from grey literature was excluded. The types of mass vaccination campaign study designs searched for in the databases included prospective, cohort and case-control. Studies reporting adverse events following immunizations via both active and passive surveillance on the ten selected vaccines either as single ingredient vaccines or in combination of two or more were included. Studies which met the inclusion criteria but failed to mention the types of AEFIs observed were excluded. Multi-centre studies which included both African and non-African countries were excluded. All studies used were ensured to have been published in the English language. Two experienced researchers in systematic review methodology independently reviewed the titles and abstracts identified and reached a consensus on the inclusion and exclusion of studies.

\section{Types of participants, interventions, comparisons and outcomes}

The participants, interventions, comparisons and outcomes (PICO) framework was adopted in the design of the systematic review protocol and served as the guide for the selection of clinical trials for the review. The potential participants in the included studies were all likely persons in various vaccine target populations (i.e infants, adolescents, pregnant women, adults and the elderly). The interventions were vaccines used either on routine basis in infant immunization schedules or mass vaccination campaigns in the prevention of infectious diseases and these included the rotavirus, yellow fever, pneumococcal, measles, rubella, tuberculosis (BCG), Haemophilus Influenza type b (Hib), polio, meningococcal and influenza A (H1N1) vaccines. Regarding the comparators to the vaccines studied, studies making relevant comparisons of vaccines against a control, such as placebo, unexposed or untreated group, or alternate vaccine formulation were included. Acceptable outcome measures included intervention efficacy, effectiveness, or safety. The major outcome evaluated was safety of the vaccines to be studied. However, studies that did not evaluate vaccine safety as a primary outcome were included if AEFI data were reported for the vaccine target populations.

\section{Assessment of methodological quality and strength of evidence}

Studies meeting the inclusion criteria were independently verified for methodological quality using the following criteria: studies with clearly defined subjects, studies with clearly defined outcomes, studies with adequate sample size, studies with responses representative of the study population, studies with high response rate, studies using validated measures, studies with measures consistent with the set aims and objectives, studies with conclusions consistent with results, studies with clear methodology and studies with clear analysis. These criteria were obtained from the Critical Appraisal Skills Program (CASP) which has been used by several authors in systematic reviews due to its reliability ${ }^{22,23}$. The McHarm instrument was used to evaluate the quality of the studies with regard to their assessment of $\mathrm{AEFIs}^{24}$. Studies that reported time of AEFI onset, AEFI severity and defined AEFI using standard, precise definitions were rated higher than those that did not. The overall strength of evidence of the vaccine safety outcome was determined by the Agency for Healthcare Research and Quality (AHRQ) guidelines ${ }^{25}$. The AHRQ guidelines prescribe 4 strength of evidence levels namely high (high confidence in AEFI reflecting the true effect), moderate (moderate confidence in the observed AEFI; further studies may alter the AEFI outcome), low (low confidence in observed AEFI; further studies more likely 
to alter the AEFI outcome) and insufficient (unavailable evidence or no confidence in observed AEFI outcome).

\section{Search strategy}

The electronic databases`searched included PUBMED, Google Scholar, Clinical trials.gov and Cochrane controlled register of trials. A three-step search strategy was utilized in the review. An initial search of PUBMED was undertaken to identify subject headings and potential keywords relevant to the systematic review. The subject headings and potential keywords in this initial search included "vaccine name" AND "clinical trials" AND "Africa" and "vaccine name", "mass vaccination campaigns" AND "Africa" and "vaccine safety" AND "Africa". A second search was carried out in Google Scholar, Clinical trials. gov and Cochrane controlled register of trials databases using these keywords, subject headings and index terms. The third search involved a hand search of the reference lists of the identified articles for additional studies that may have been missed during the systematic search. The reference lists selected for full text review were searched for the relevance of the articles. The search considered articles published from $1^{\text {st }}$ January 2000 to $31^{\text {st }}$ December 2015 only. Literature search was done on the $27^{\text {th }}$ of May 2016 using the proposed structure according to the preferred reporting items for systematic reviews and meta-analyses ${ }^{26}$.

\section{Screening and data extraction}

The initial screening of published papers was conducted by one reviewer; a two reviewer system was employed throughout the rest of the review workflow. Search results were imported into Mendeley referencing software and one reviewer screened titles and abstracts of the published papers. Articles and titles with no bearing to the review were discarded. Consensus and discussion with a second reviewer was the means of resolving uncertainty during the screening process regarding inclusion and exclusion of studies. A data extraction sheet was used to collect relevant information from the included studies (Appendix 1). This information included the details of the author, the aims and objectives of the study, the research setting, the research participants and sample size, the study design used and vaccine safety related information reported in the studies. Common Terminology Criteria for Adverse Events classification system (Appendix 2) was used to characterize the severity of AEFIs reported in the studies 27 . Tables were used to present the summary of extracted data.

\section{Quality analysis (meta-analysis) of included studies}

Data analysis was planned to provide a detailed overview of the heterogeneity among AEFI definitions and reporting patterns in the included studies based on the WHO / CIOMS definitions. The analysis performed after ensuring homogeneity of studies included:

a. Assessment of whether AEFIs are defined or reported in the included studies.

b. Assessment of studies that reported AEFI severity and defined AEFI using standard and precise definitions

c. Characterization of consistency in AEFI names and definitions.

d. Ranking of reported AEFIs into minor and serious based on WHO/CIOMS definitions.

e. Assessing the strength of evidence of AEFI outcomes

\section{Results}

\section{Results of literature search}

After the literature search, 3,989 studies were identified and recorded. The electronic databases (PUBMED, Google Scholar, Clinical trials.gov and Cochrane controlled register of trials) search retrieved 3,932 studies whereas 57 were obtained from the reference list of the included studies. Out of these articles, 3,840 duplicates were identified and removed, leaving 149 studies to be assessed. The titles and abstracts of the remaining studies were closely reviewed against the inclusion and exclusion criteria and 101 studies were excluded. An additional 36 were excluded after scrutinizing the text against the inclusion and exclusion criteria. The entire process led to $12 \mathrm{good}$ quality studies that met the inclusion criteria as presented in the PRISMA flow diagram in Fig 1 which is based on the Moher et al, 2009 study $^{27}$. 


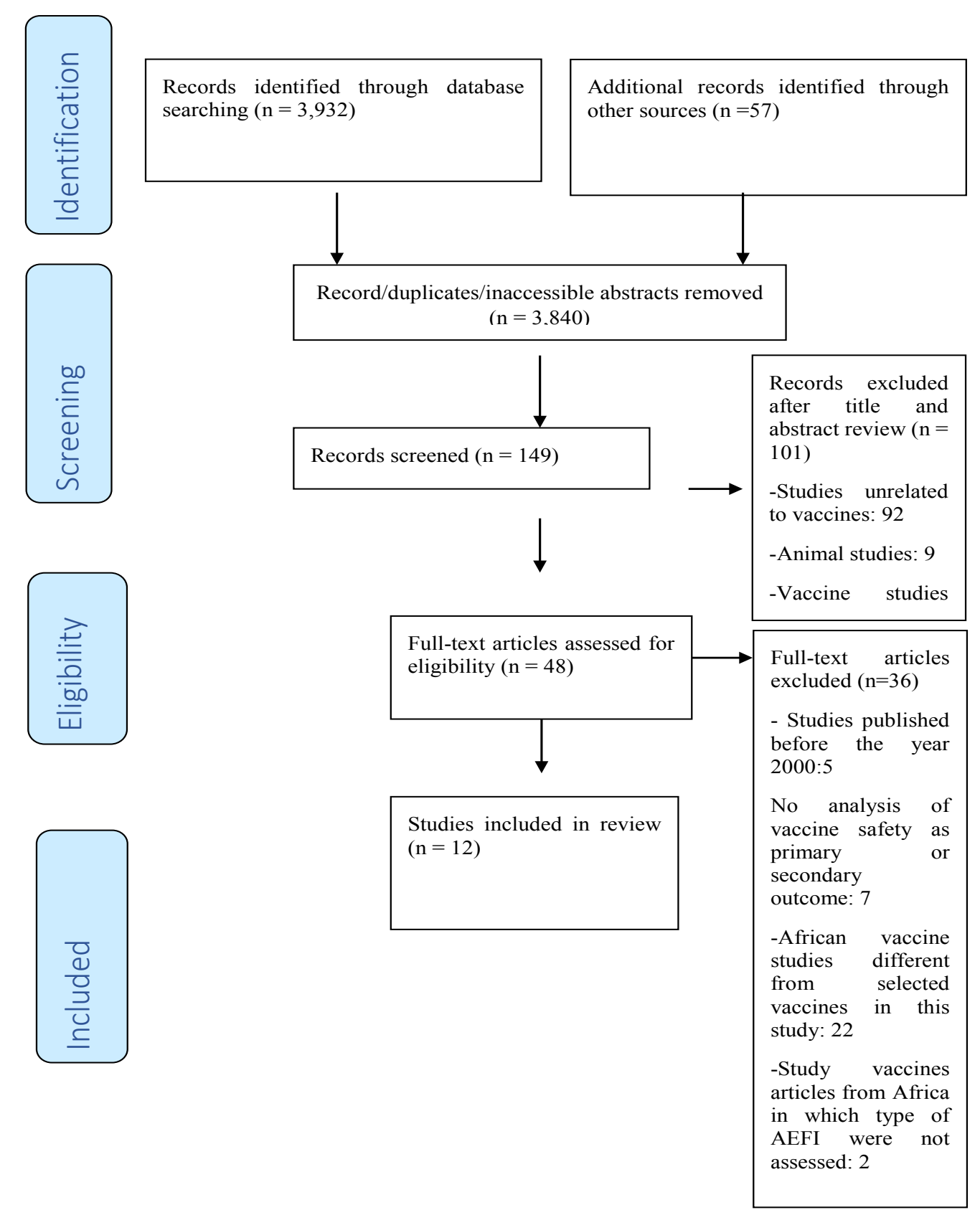

Fig 1: PRISMA diagram for the review

\section{Characteristics of included studies}

There was one study on Haemophilus influenza type b vaccine $^{28}$, two studies on influenza A (H1N1) vaccine ${ }^{29,30}$, one study on measles-rubella vaccine ${ }^{31}$, one study on meningococcal vaccine ${ }^{32}$, one study on oral polio vaccine ${ }^{33}$, two studies on pneumococcal vaccine ${ }^{34,35}$, one study on rotavirus vaccine ${ }^{36}$, two BCG vaccine studies ${ }^{37,38}$ and one yellow fever vaccine study ${ }^{39}$. With the exception of only one study which assessed two vaccines (Measles-Rubella) in one formulation ${ }^{31}$, all the remaining studies assessed vaccines containing only one active ingredient. Vaccinees in the included studies were neonates, infants, children, adolescents and adults. All studies included in this review were published between 2001 and 2013 and were clinical trials, prospective and cohort studies. No case-control study was retrieved from the literature search. The study with the least number of participants was the phase 1 study in South Africa in which 24 healthvolunteers were recruited ${ }^{37}$. 
Another characteristic of the studies used in the review was that participants from all parts of Africa i.e. West, East, Central, North and Southern Africa were represented. The West African countries were Ghana ${ }^{29,36}$, Togo ${ }^{39}$, Benin $^{39}$, Mali $^{32,36,39}$, Senegal ${ }^{12,39}$, Liberia ${ }^{39}$, Guinea ${ }^{39}$, Sierra Leone $^{39}$ and Gambia ${ }^{32,34}$. The east African country was Kenya $^{39}$. The central African countries were Cameroon ${ }^{39}$ and Democratic Republic of Congo ${ }^{33}$. The North African countries were Egypt ${ }^{31}$ and Tunisia ${ }^{30}$ whereas South Africa was the only Southern African country ${ }^{28,35,37,38}$. Three of the studies ${ }^{32,36,39}$ were multi-centre in design whereas the remaining nine were conducted in only one country. Further details of the location, study objective, participant characteristics, study design and AEFI outcome are presented in Table 1.

Table 1: Location, study objective, participant characteristics, study design and AEFI outcome of studies

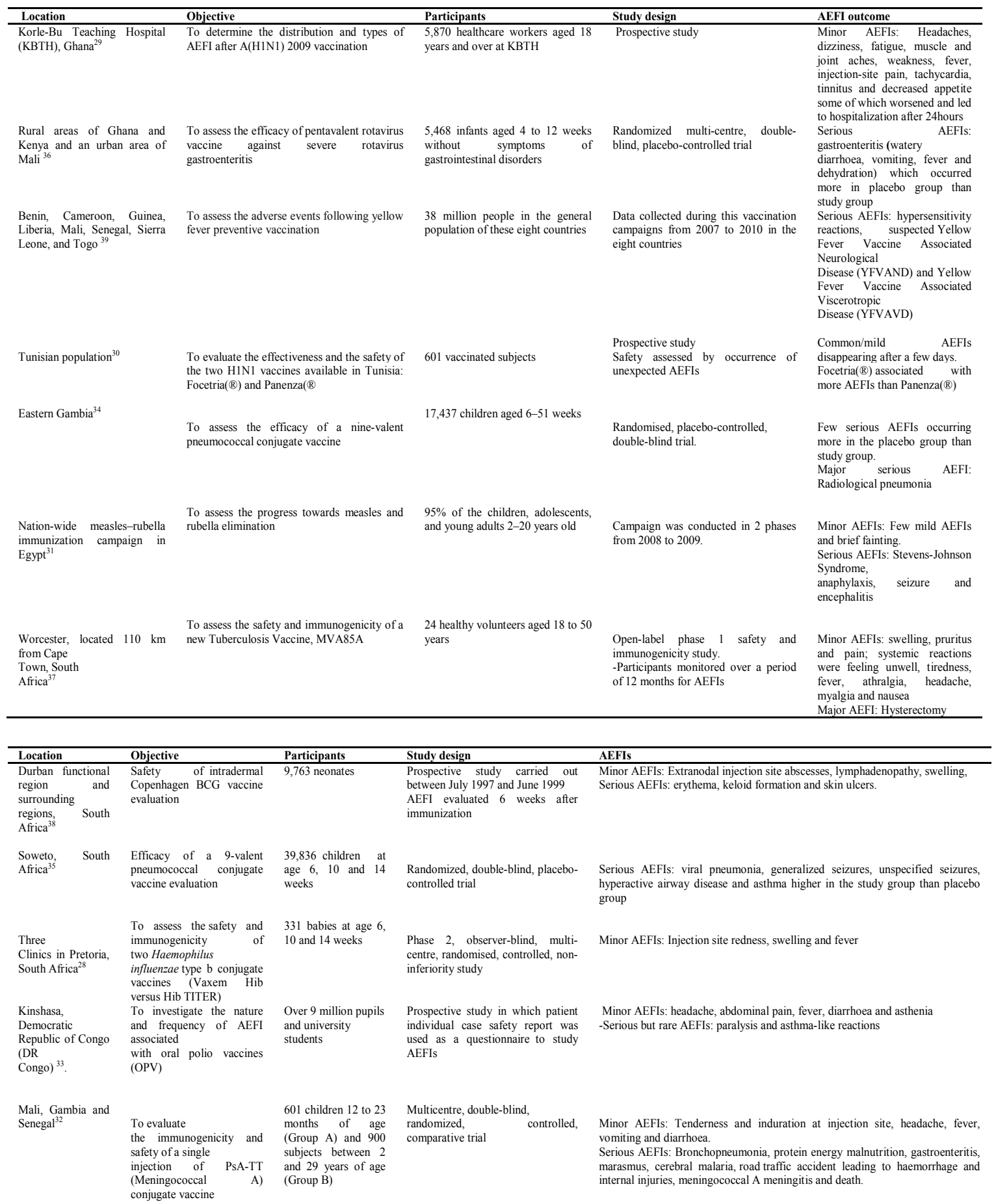


Meta-Analysis (Strength of evidence of AEFI out- The strength of AEFI outcomes based on AHRQ guidecomes) lines and reasons are summarized in Table 2.

\begin{tabular}{|c|c|c|}
\hline Vaccine & $\begin{array}{l}\text { Strength } \\
\text { of } \quad \text { evidence of } \\
\text { AEFI }\end{array}$ & Reason \\
\hline $\begin{array}{l}\text { Haemophilus } \\
\text { Influenza type b }\end{array}$ & Moderate & Only one study reporting AEFIs was identified ${ }^{28}$. \\
\hline Influenza A (H1N1) & Moderate & $\begin{array}{l}\text { Two studies were identified but only one study }{ }^{29} \text {. mentioned } \\
\text { the specific AEFIs. The specific AEFIs were not mentioned in } \\
\text { the other study }{ }^{30} \text {. }\end{array}$ \\
\hline Measles-Rubella & Moderate & $\begin{array}{l}\text { High confidence in AEFI outcome because of high sample size } \\
\text { but only one study reported these AEFIs }{ }^{31} \text {. }\end{array}$ \\
\hline Meningococcal & Moderate & $\begin{array}{l}\text { High confidence in AEFI outcome because of long term follow- } \\
\text { up of study participants. However, only one study reported these } \\
\text { outcomes making strength of evidence moderate }{ }^{32} \text {. Further } \\
\text { studies are needed for comparison of AEFI outcomes with that in } \\
\text { current study. }\end{array}$ \\
\hline Oral Polio & Moderate & $\begin{array}{l}\text { High confidence in AEFI outcome because of high sample size } \\
\text { but only one study was identified reporting these } \\
\text { AEFIs }{ }^{33} \text {. Further studies are needed for comparison of AEFI } \\
\text { outcomes with that in current study. }\end{array}$ \\
\hline Pneumococcal & Moderate & $\begin{array}{l}\text { Two studies identified }{ }^{34,35} \text { but only few minor AEFIs occurred in } \\
\text { both studies. Further studies are needed for clarity. }\end{array}$ \\
\hline Rotavirus & Moderate & $\begin{array}{l}\text { Only one study identified with AEFIs. There is the need for } \\
\text { further studies for AEFI comparison }{ }^{36} \text {. }\end{array}$ \\
\hline Tuberculosis (BCG) & High & Two studies identified with similar AEFIs ${ }^{37,38}$. \\
\hline Yellow fever & Moderate & $\begin{array}{l}\text { Only one study identified with the AEFIs observed }{ }^{39} \text {. More } \\
\text { studies are needed for clarity }\end{array}$ \\
\hline
\end{tabular}

\section{Discussion}

Twenty four serious AEFIs were identified in this review and included Stevens-Johnson Syndrome, anaphylaxis, seizure, encephalitis, bronchopneumonia, protein energy malnutrition, gastroenteritis, marasmus, cerebral malaria, road traffic accident leading to haemorrhage and internal injuries, meningococcal A meningitis, paralysis, asthma and asthma-like reactions, radiological pneumonia, viral pneumonia, generalized seizures, unspecified seizures, hyperactive airway disease, elective hysterectomy, hypersensitivity reactions, suspected yellow fever vaccine associated neurological disease (YFVAND), suspected yellow fever vaccine associated viscerotropic disease (YFVAVD), hospitalization and death. The serious AEFIs associated with deaths were linked to the meningococcal vaccine. Studies from other regions of the world recorded no fatal AEFIs association with the meningococcal vaccine as recorded in this review indicating that further research on the meningococcal vaccine may be needed to confirm the findings in this study. With the exception of the Hib ad H1N1 vaccine, all other vaccines were associated with serious AEFIs. The finding with the H1N1 vaccine is consistent with findings from other studies which found no serious AEFI following its administration confirming its safety ${ }^{40,41}$.

Active forms of organisms used in vaccine manufacture could cause serious AEFIs. For instance OPV and pneumococcal vaccine were associated with paralysis and radiological pneumonia respectively. Even though, the inactivated polio vaccine (IPV) which is being used in many developed countries is associated with paralytic poliomyelitis to a far lesser extent ${ }^{42}$, it is also associated with allergic reactions such as food allergy and the asthma-like reactions identified in this review ${ }^{43}$. However, because the OPV associated paralysis and asthma-like reactions were rare, the use of the OPV may be continued in routine immunization. However, further comparative studies of OPV and IPV in African settings will be beneficial in informing policy as to whether to switch to the IPV or not.

Rotavirus vaccine was associated with serious gastroenteritis. A systematic review on the safety of routinely used vaccines conducted in the United States of America (USA) found that rotavirus vaccine was not associated 
with gastroenteritis but rather, cough, runny nose and irritability. ${ }^{44}$ Moreover, a study conducted in Australia also observed intussusception in children aged 1 to 21 days after immunization with $\operatorname{RotaTek}\left({ }^{\circledR}\right.$, a popular brand of rotavirus vaccine) and this was consistent with the finding in a study in Mexico after immunization with another brand, Rotarix $\left({ }^{\circledR 4}\right.$. These findings are related to concerns which led to the withdrawal of the rotavirus vaccine, Rotashield ( $($ from the USA market demonstrating the need to study the safety of the rotavirus vaccine extensively ${ }^{46}$. The serious AEFIs in the yellow fever multi-centre study, yellow fever vaccine associated neurological disease (YFVAND) and suspected yellow fever vaccine associated viscerotropic disease (YFVAVD) have been observed in other studies and must be noted carefully by vaccine program managers and Expanded Program on Immunization managers ${ }^{47,48}$. Due to these serious AEFIs, Lindsey et al., 2008 in their study concluded that yellow fever vaccination should be limited to persons travelling to areas where the risk of yellow fever is expected to exceed the risk of serious adverse events after vaccination, or if not medically contraindicated, where national regulations require proof of vaccination to prevent introduction of yellow fever ${ }^{48}$.

Minor AEFIs are usually local reactions at the site of injection and others that resolve within a few days after immunization posing little or no danger to the vaccinee. Twenty-three minor AEFIs were identified in the review and included headaches, dizziness, muscle and joint aches, weakness, fever, injection-site pain, diarrhoea, redness, tenderness and induration of site of injection, swelling, abscesses, brief fainting, abdominal pain, tachycardia, tinnitus, decreased appetite, asthenia, diarrhoea, pruritus, erythema, lymphadenopathy, keloid formation and ulcers of the skin. Besides the active ingredients used in the preparation of vaccines, certain additives are also included to increase their potency and preserve them. These include but not limited to suspending agents (e.g. water and saline), preservatives (e.g. thimerosal), stabilizers (e.g. sorbitol and hydrolyzed gelatin), adjuvants (mainly aluminum salts), residuals in the growth medium and antibiotics (e.g. neomycin and streptomycin). ${ }^{49}$ Certain individuals may react to the active vaccine products or these additives leading to AEFIs. It is therefore imperative to document any such previous reactions to prevent their re-occurrence in future.
It is important to note that AEFIs are not necessarily caused by vaccine products but several other factors necessitating the need for associations and causality assessments on AEFIs ${ }^{12,13,50}$ in order to make an informed decision as to whether to withdraw a vaccine or not. In this review, the strength of association between AEFIs and vaccine was high for Tuberculosis vaccine and moderate for all other vaccines.

\section{Study limitations}

The major limitation of this study was the inclusion of studies in which AEFIs were reported passively due to scanty data on active AEFI reporting studies. Even though passive AEFI are useful, they can sometimes be subjective and unreliable. Moreover, participants in the included studies were not followed up over a long period of time to adequately identify rare and late occurring AEFIs. Although clinical trials are rigorous in nature, they also do not have large sample sizes to adequately identify rare AEFIs. It will therefore be more appropriate to use pharmacoepidemiological study designs such as cohort event monitoring in which participants are monitored over a long period of time with active reporting mechanisms to establish better associations between vaccines and AEFIs.

\section{Conclusion}

Overall, all the vaccines studied were generally well tolerated. The strength of association between AEFIs and vaccine was high for tuberculosis (BCG) vaccine and moderate for all other vaccines.

\section{Conflict of interest}

The authors declare that this study is not associated with any conflict of interest.

\section{Funding sources}

Research reported in this publication was supported by the Fogarty International Centre (FIC), National Institutes of Health Common Fund, Office of Strategic Coordination, Office of the Director (NIH CF/OSC/ OD), Office of AIDS Research, Office of the Director $(\mathrm{OAR} / \mathrm{OD} / \mathrm{NIH})$, National Institute of Mental Health of the NIH (NIMH/NIH) under Award Number D43TW010131. The content is solely the responsibility of the authors and does not necessarily represent the official views of the NIH. 


\section{Acknowledgements}

We wish to acknowledge the contribution of Dr. Didier Nzolo of the national pharmacovigilance centre of the Democratic Republic of Congo for his immense contribution on the current situation of vaccine pharmacovigilance in the African sub-region and the WHO Collaborating centre for Advocacy and training in Pharmacovigilance, Ghana, for in-depth material on vaccine pharmacovigilance on the global landscape.

\section{References}

1. Greenwood B. The contribution of vaccination to global health: past, present and future. Philosophical Transactions of the Royal Society B: Biological Sciences 2014; 369(1645): 20130433. http://doi.org/10.1098/rstb.2013.0433

2. Roeder P, Mariner J, Kock R. Rinderpest: the veterinary perspective on eradication. Philosophical Transactions of the Royal Society B 2013; 368: 20120139. doi:10.1098/ rstb.2012.0139

3. Weiss RA \& Esparza J. The prevention and eradication of smallpox: a commentary on Sloane (1755) "An account of inoculation." Philosophical Transactions of the Royal Society B: Biological Sciences 2015; 370(1666): 20140378. http://doi.org/10.1098/rstb.2014.0378

4. Youde J. Cattle scourge no more. The eradication of rinderpest and its lessons for global health campaigns. Politics and the Life Sciences 2013; 32: 43-57. (doi:10.2990/32_1_43) 5. Larson HJ, Cooper LZ, Eskola J, Katz SL, Ratzan S. Addressing the vaccine confidence gap. The Lancet 2011; 378: 526-535 PubMed.

6. Miller ER, Moro PL, Cano M \& Shimabukuro T. Deaths following vaccination: What does the evidence show? Vaccine 2015; 33(29): 3288-3292. doi:10.1016/j. vaccine.2015.05.023

7. Clemmons NS, Gastanaduy PA, Fiebelkorn AP, Redd SB, Wallace GS. Centres for Disease Control and Prevention [CDC], (2015). Measles-United States, January 4 April 2, 2015. Morbidity and Mortality Weekly Report 2015; 64(14): 373-376. [PubMed: 25879894]

8. Huff EA. Measles vaccines kill more people than measles, CDC data proves, Global Research; 2015. Available at http://www.globalresearch.ca/measles-vaccines-killmore-people-than-measles-cdc-data-proves/5429736

9. Shilhavy B. Zero US measles deaths in 10 years, but over 100 measles vaccine deaths reported; 2015. Health Impact News. Available at http://healthimpactnews. com/2015/zero-u-s-measles-deaths-in-10-years-butover-100-measles-vaccine-deaths-reported/
10. World Health Organization. Definition and application of terms for vaccine Pharmacovigilance Report of CIOMS/WHO Working Group on Vaccine Pharmacovigilance; 2012. Geneva, Council for International Organizations of Medical Sciences.

11. Report of CIOMS/WHO Working Group: Definition and Application of Terms for Vaccine Pharmacovigilance, 32. Available at:http://www.who.int/vaccine_safety/initiative/tools/CIOMS_report_WG_vaccine.pdf 12. Global Advisory Committee on Vaccine Safety and WHO Secretariat (2009). Global safety of vaccines: Strengthening systems for monitoring, management and the role of GACVS. Expert Review Vaccine 2009; 8(6): 705716, PMID: 19485752, DOI: 10.1586/erv.09.40

13. World Health Organization. Developing a national system for dealing with adverse events following immunization; 2013. Available at: http://www.scielosp.org/ scielo.php?pid $=$ S0042-96862000000200004\&script=sci., 14. Dreskin SC, Halsey NA, Kelso JM, Wood RA, Hummell DS, Edwards KM et al. International Consensus (ICON): allergic reactions to vaccines. The World Allergy Organization Journal 2016; 9(1), 32. Available at: http:// doi.org/10.1186/s40413-016-0120-5

15. Halsey NA. The science of evaluation of adverse events associated with vaccination. Seminars Pediatric Infectious Diseases 2002; 13(3):205-214. doi: 10.1053/ spid.2002.125864

16. Cano M \& Lewis P. Bell's palsy Cases Following Administration of Influenza A (H1N1), Monovalent Vaccine Reported to the Vaccine Adverse Event Reporting System (VAERS). Journal of Vaccines \& Vaccination 2009; 03 (02).

17. Delage G. Rotavirus vaccine withdrawal in the United States: The role of post-marketing surveillance. Canadian Journal of Infectious Diseases 2000; 11(1): 10-11

18. World Health Organization. Western Pacific Region, Immunization Safety Surveillance, Guidelines for immunization program managers on surveillance of Adverse Event Following Immunization, Second Edition, 2013. Available at:http://www.wpro.who.int/topics/immunization_safety/ImmunizationSafetySurveillance.pdf

19. Craddock S. Drug partnerships and global practices. Health Place 2012;18:481-489 PubMed

20. Idoko OT, Kochhar S, Agbenyega TE, Ogutu B, \& Ota MOC. Impact, Challenges, and Future Projections of Vaccine Trials in Africa. The American Journal of Tropical Medicine and Hygiene 2013; 88(3): 414-419. http://doi. org/10.4269/ajtmh.12-0576 
21. Centres for Disease Prevention and Control. Vaccines and Preventable Diseases; 2018 Available at: https:// www.cdc.gov/vaccines/vpd/vaccines-list.html

22. Critical Appraisal Skills Programme. Public Health Resource Unit, Institute of Health Science, Oxford; 2013. Available at: http://www.casp-uk.net/\#!checklists/cb36 23. Harrison JK, Reid J, Quinn TJ \& Shenkin SD. Using quality assessment tools to critically appraise ageing research: a guide for clinicians. Age and Ageing 2017; 46(3):359-365. http://doi.org/10.1093/ageing/afw223

24. Santaguida PL, Raina P. The Development of the McHarm Quality Assessment Scale for adverse events: Delphi Consensus on important criteria for evaluating harms. 2008. Available at: http://hiru.mcmaster.ca/ epc/ mcharm.pdf.

25. Owens DK, Lohr KN, Atkins D, Treadwell JR, Reston JT, Bass EB, et al. AHRQ series paper 5: grading the strength of a body of evidence when comparing medical interventions - agency for healthcare research and quality and the effective health-care program. Journal of Clinical Epidemiology 2010; 63(5): 513-523.

26. Moher D, Liberati A, Tetzlaff J, Altman DG \& The PRISMA Group. Preferred Reporting Items for Systematic Reviews and Meta-Analyses: The PRISMA Statement. PLoS Med 2009; 6(7): e1000097. doi:10.1371/journal.pmed1000097

27. US Department of Health and Human Services. Common Terminology Criteria for Adverse Events (CTCAE). 2009. Version 4.0.

28. Matjila MJ, Phohu TC, Banzhoff A, Viviani S, Hoosen AA, Bianchini M, et al. Safety and immunogenicity of two Haemophilus influenzae type B conjugate vaccines, South African Medical Journal 2004; 94:43-46 PubMed .

29. Ankrah DN, Mantel-Teeuwisse AK, De Bruin ML, Amoo PK, Ofei-Palm CN, Agyepong I, et al. (2013). Incidence of adverse events among healthcare workers following H1N1 Mass immunization in Ghana: a prospective study. Drug Safety 2013; 36(4):259-266.

30. Chaabane A, Aouam K, Fredj NB, Toumi A, Braham $\mathrm{D}$, Boughattas $\mathrm{N}$, et al. H1N1 influenza vaccines in Tunisia: efficiency and safety. Therapie 2011; 66(3):281-289.

31. El Sayed N, Kandeel N, Barakat I, Moussa I, Alexander Jr JP, Naouri B, et al. Progress toward Measles and Rubella elimination in Egypt. Journal of Infectious Diseases 2011; 204: S318-S324.

32. Sow SO, Okoko BJ, Diallo A, Viviani S, Borrow R, Carlone $\mathrm{G}$, et al. Immunogenicity and safety of a menin- gococcal A conjugate vaccine in Africans. New England Journal of Medicine 2011;16(364): 2293-2304

33. Nzolo D, Aloni MN, Ngamasata TM, Luemba BM, Marfeza SB, Ekila MB, et al. Adverse events following immunization with oral poliovirus in Kinshasa, Democratic Republic of Congo: preliminary results. Pathogens and Global Health 2013;107(7): 381-384.

34. Cutts FT, Zaman SMA, Enwere G, Jaffar S, Levine OS, Okoko JB, et al. Greenwood, B.M.(2005). Efficacy of nine-valent pneumococcal conjugate vaccine against pneumonia and invasive pneumococcal disease in the Gambia: randomised, double-blind, placebo controlled trial. Lancet 2005; 365:1139-1146.

35. Klugman KP, Madhi SA, Huebner RE, Kohberger R, Mbelle N\& Pierce N. A trial of a 9-valent pneumococcal conjugate vaccine in children with and those without HIV infection. New England Journal of Medicine 2003; (349): 1341-1348.

36. Armah GE, Sow SO, Breiman RF, Dallas MJ, Tapia $\mathrm{MD}$, Feikin DR et al. Efficacy of pentavalent rotavirus vaccine against severe rotavirus gastroenteritis in infants in developing countries in sub-Saharan Africa: a randomised, double-blind, placebo-controlled trial. Lancet 2010; 21(376): 606-614.

37. Hawkridge T, Scriba TJ, Gelderbloem S, Smit E, Tameris M, Moyo S, et al. Safety and Immunogenicity of a New Tuberculosis Vaccine, MVA85A, in Healthy Adults in South Africa. The Journal of Infectious Diseases 2008;198: $544-552$

38. Jeena PM, Chhagan MK, Topley J \& Coovadia HM. Safety of the intradermal Copenhagen 1331 BCG vaccine in neonates in Durban, South Africa, Bulletin of the World Health Organization 2001; 79: 337-343.

39. Breugelmans JG, Lewis RF, Agbenu E, Veit O, Jackson D, Domingo C...Yactayo, S. Adverse events following yellow fever preventive vaccination campaigns in eight African countries from 2007 to 2010. Vaccine 2013; 31(14): 1819-1829.

40. Englund JA, Walter E, Black S, Blatter M, Nyberg J, Ruben FL, et al. Safety and immunogenicity of trivalent inactivated influenza vaccine in infants: a randomized double-blind placebo-controlled study. Pediatric Infectious Diseases Journal 2010; 29 (2): 105-110

41. Mallory RM, Malkin E, Ambrose CS, Bellamy T, Shi L, Yi T, et al. Safety and immunogenicity following administration of a live, attenuated Monovalent 2009 H1N1 influenza vaccine to children and adults in two randomized controlled trials. PLoS One 2010; 5(10): e13755. 
42. Platt LP, Estívariz CF, \& Sutter RW. Vaccine-Associated Paralytic Poliomyelitis: A Review of the Epidemiology and Estimation of the Global Burden. The Journal of Infectious Diseases 2014; 210 (suppl_1): S380-S389. Available at: https://doi.org/10.1093/infdis/jiu184

43. Grüber C, Warner J, Hill D, Bauchau V \& EPAAC Study Group. Early atopic disease and early childhood immunization-is there a link? Allergy 2008; 63(11):1464 1472

44. Maglione MA, Das L, Raeen L, Smith A, Chari R, Newberry S, et al. Safety of Vaccines Used for Routine Immunization of US Children: A Systematic Review. Pediatrics 2014; 134: 1-13 PubMed .

45. Velázquez FR, Colindres RE, Grajales C, Hernandez MT, Mercadillo MG, Torres FJ, et al. Postmarketing surveillance of intussusception following mass introduction of the attenuated human rotavirus vaccine in Mexico. $\mathrm{Pe}$ diatric Infectious Diseases Journal 2012; 31(7): 736-744.

46. Cano M \& Lewis P. Bell's palsy Cases Following Administration of Influenza A (H1N1), Monovalent Vac- cine Reported to the Vaccine Adverse Event Reporting System (VAERS). Journal of Vaccines \& Vaccination 2009; $03(02)$

47. Belsher JL, Gaya P, Brinton M, DellaValla J, Ridenour $\mathrm{R}$, Perelygin A, et al. Fatal multi-organ failure due to yellow fever vaccine-associated viscerotropic disease. Vaccine 2007; 25: 8480-8485 PubMed .

48. Lindsey NP, Schroederb BA, Millerc ER, Braun MM, Hinckley AF, Marano N, et al. Adverse event reports following yellow fever vaccination. Vaccine 26; 2008: $6077-$ 6082.

49. US Food and Drugs Administration. Vaccines, Blood and Biologics, Common Ingredients in US licensed vaccines. 2014. Available at: https://www.fda.gov/BiologicsBloodVaccines/SafetyAvailability/VaccineSafety/ ucm187810.htm

50. Beirne PV, Hennessy S, Cadogan SL, Shiely F, Fitzgerald T, MacLeod F. Needle size for vaccination procedures in children and adolescents. Cochrane Database Systematic Review 2015; 18 (6):CD010720. doi: 10.1002/14651858. CD010720.pub2 